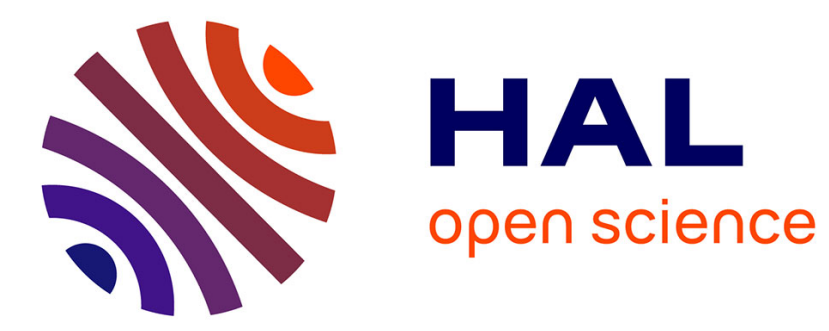

\title{
Territoire et lien social: heurs et malheurs de la gestion territorialisée de l'exclusion
}

Raymonde Séchet

\section{To cite this version:}

Raymonde Séchet. Territoire et lien social: heurs et malheurs de la gestion territorialisée de l'exclusion. Travaux de l'Institut de Géographie de Reims, 1995, 91-92, pp.23-38. halshs-00314011

\section{HAL Id: halshs-00314011 https://shs.hal.science/halshs-00314011}

Submitted on 30 Sep 2008

HAL is a multi-disciplinary open access archive for the deposit and dissemination of scientific research documents, whether they are published or not. The documents may come from teaching and research institutions in France or abroad, or from public or private research centers.
L'archive ouverte pluridisciplinaire HAL, est destinée au dépôt et à la diffusion de documents scientifiques de niveau recherche, publiés ou non, émanant des établissements d'enseignement et de recherche français ou étrangers, des laboratoires publics ou privés. 


\title{
TERRITOIRE ET LIEN SOCIAL
}

\author{
Heurs et malheurs de la gestion \\ territorialisée de l'exclusion
}

Raymonde
SECHET

URA CNRS 915,

Université Rennes 2

\begin{abstract}
Mots-clés : Territoire - Pauvreté - Exclusion - Régulation sociale - Intervention sociale.
Résumé - Le grand retour de la pauvreté absolue, tant dans les esprits que dans la rue, et la réorientation de l'action sociale de la réduction des inégalités vers la lutte contre l'exclusion ont entraîné la mise en place d'un nouveau modèle d'intervention sur le social. Les nouveaux dispositifs créés en France pour maintenir le lien social placent le territoire au coeur de la régulation sociale. Le passage à un mode de gestion sécuritaire redonne toute son importance à la question du rôle de l'espace dans le contrôle social. Cependant, la territorialisation des dispositifs sociaux ne va pas sans effets pervers car la combinaison encadrement / autonomie communautaire constitutive de la régulation post-keynésienne peut déboucher sur l'émergence de contre-territoires et sur l'ethnicisation des banlieues.
\end{abstract}

Territory and social ties : good fortune and misfortune in the territorial management of exclusion.

Key-words : Territory - Poverty - Exclusion - Social Regulation - Social Intervention

Abstract - The return of absolute poverty, in minds as well as in the streets, and the reorientation of the social action meant to reduce inequalities towards exlusion, have, as a consequence, set up a new model of intervention in the social field. The new measures implemented in France to maintain social relationships put the territory at the heart of social regulation. The passage to security management reveals once more the importance of the role of space in social control. However, territorialization of social measures is not without effects, for the combination of control and autonomy of the community, which constitutes post-keynesian regulation, can induce the emergence of counter-territories and the ethnic division of suburbs. 
Les écrits de J. K. Galbraith ${ }^{1}$ et M. Harrington ${ }^{2}$ aux Etats-Unis dans les années soixante, ceux de R. Lenoir ${ }^{3}$ et L. Stoléru ${ }^{4}$ en France au début des années soixante-dix, ont mis fin au mythe, entretenu par le paradigme modernisateur, de la disparition de la pauvreté dans les pays riches. Par fidélité à la tradition positiviste de "l'état des lieux", les géographes ont alors plus spontanément pensé la pauvreté comme addition de situations individuelles que comme système de représentations guidant des politiques. Leurs travaux ont donné une dimension spatiale à la problématique du seuil de pauvreté. D'abord clairement formulée par John Galbraith ${ }^{5}$, la notion de poches ou d'îlots de pauvreté a ensuite été reprise par Richard Peet qui a étudié des espaces de pauvreté, ruraux ou urbains, délimités par la concentration spatiale de situations individuelles marquées par le dénuement, un cumul de handicaps aboutissant à un cercle vicieux de la pauvreté, des comportements relevant de la culture de pauvreté avec, notamment, un enracinement territorial très fort ${ }^{6}$.

Globalement, dans les années soixante-dix, surtout en France, la pauvreté n'apparaît pas comme un problème différent des inégalités sociales. La production de pauvreté est pensée en relation avec les processus économiques producteurs d'inégalités, ... et producteurs d'espace. D'ailleurs, pour les chercheurs en analyse spatiale, "les couples richesse-pauvreté et centre-périphérie sont liés dans la nature de leurs relations comme dans la source du phénomène"7. Les modèles d'analyse du sousdéveloppement et des inégalités spatiales laissent peu de place au sociétal et au symbolique. Toutefois, des géographes travaillant sur la marginalité sont allés au-delà du double déterminisme des lieux et des rapports de production pour étudier autant sa construction sociale que l'objet lui-même et pour poser avec plus de complexité la question des relations entre pauvreté, espace et territoires : "Quelle fonction joue l'espace dans le processus d'exclusion et quel rôle lui fait-on jouer ? (...) Réaliser une géographie de la marginalité, c'est s'interroger sur les manières dont les systèmes dominants ou "centraux" utilisent l'espace pour exclure certains groupes sociaux. C'est aussi se demander comment ces groupes

\footnotetext{
$1^{1}$ J. K. Galbraith, L'ère de l'opulence, Paris, Calmann-Lévy, 1961, 332 p. Trad. de The affluent society, Boston, Houghton Mifflin C ${ }^{\circ}$, 1968.

${ }^{2}$ M. Harrington, L'autre Amérique. La pauvreté aux Etats-Unis, Gallimard, coll. Témoins, 1967, 289 p. Trad. de The other America : Poverty in the United States, Baltimore, Penguin Books, 1963.

${ }^{3} \mathrm{R}$. Lenoir, Les exclus. Un français sur dix, Editions du Seuil, 1974, $171 \mathrm{p}$.

${ }_{5}^{4}$ L. Stoleru, Vaincre la pauvreté dans les pays riches, Flammarion, coll. Champs, 1977, $319 \mathrm{p}$.

5 "On peut considérer que la pauvreté moderne est susceptible de se ranger dans deux grandes catégories. Il existe d'abord ce qu'on pourrait appeler "les cas d'espèce". Ils se rencontrent dans toute communauté, rurale ou urbaine, quelle que soit la prospérité de la communauté et de l'époque envisagées. (...) Il y a ensuite ce qu'on pourrait qualifier de pauvreté "localisée", celle qui se manifeste sous la forme d'un "îlot de pauvreté". Dans cet îlot tout le monde ou presque est pauvre. Dans ce cas-là, il n'est évidemment pas facile d'expliquer la situation par une insuffisance personnelle. (...) Pour une raison ou une autre, les gens de cet îlot ont été frustrés par leur milieu environnant." J. K. Galbraith, L'ère de l'opulence, op. cité, p. 302-303.

${ }^{6}$ R. Peet, "Outline for a second-year course on the socioeconomic geography of american poverty", Antipode, vol. $2, \mathrm{n}^{\circ} 2$, décembre 1970, pp. 1-33.

${ }^{7}$ P. Aydalot, Dynamique spatiale et développement inégal, Economica, 1976, p. 5.
} 
réagissent et tirent parti - ou non - de leur territoire pour s'enfermer ou sortir de leur position marginale"1.

La perception de la pauvreté depuis le milieu des années quatre-vingt, tant en France qu'ailleurs, est très marquée par le recul de la problématique inégalitaire et par la mise en avant des nouveaux pauvres, c'est-à-dire par le grand retour, sans nul doute dans la réalité, mais encore plus dans les discours, de la pauvreté absolue et de l'exclusion... La question de la mesure de la pauvreté est revenue au premier plan des travaux des économistes... très écoutés, et très sollicités par les décideurs ayant en charge la lutte contre la pauvreté. Dans le même temps, les débats autour de 1'urban underclass ${ }^{2}$ ont donné une nouvelle popularité aux notions de culture et d'îlots de pauvreté : "Le ghetto d'aujourd'hui est composé presque exclusivement des segments les plus défavorisés de la communauté noire, groupement hétérogène d'exclus du système professionnel américain : individus sans qualification, chômeurs de longue durée, inactifs, délinquants, hommes ou femmes se livrant à des activités anormales, familles confrontées à de longues périodes de pauvreté qui dépendent de l'aide sociale. Telles sont les populations auxquelles se réfere la notion de sous-prolétariat urbain [underclass]. Il s'agit d'une réalité sociale dont ne rend pas compte la dénomination plus standard de classe inférieure [lower class $]^{\text {"3 }}$.

Le grand retour de la pauvreté absolue, tant dans les esprits que dans la rue, et la réorientation des actions prioritaires de la réduction des inégalités vers la lutte contre l'exclusion ont entraîné un renouvellement des politiques sociales et des méthodes d'intervention sociale. Le recours au territoire local, l'essor des politiques transversales fortement territorialisées dans lesquelles, en agissant sur des espaces précis, ce sont des populations localisées que l'on veut traiter ${ }^{4}$, est devenu un phénomène si général qu'il semble bien correspondre à la mise en oeuvre d'une régulation postkeynésienne. Quand "la reconstitution d'un espace maîtrisé, comme forme spatiale de compromis négociés, reste le seul objectif valable pour les forces progressistes dans le domaine urbain" ${ }^{5}$ afin de parvenir à une gestion plus efficace des sociétés, les acteurs politiques recourent aux idéologies spatiales supposées favoriser le retour à la communauté, "la Gemeinschaft, chaude et hospitalière", au détriment de la société, "la Gesellschaft, froide et calculatrice" 6 .

\footnotetext{
1 G. Courade, "Jalons pour une géographie de la marginalité en Afrique noire", L'espace géographique, 1985, n², pp. 139-150.

2 L'ouvrage de William J. Wilson, The Truly Disadvantaged, paru en 1987, est devenu le point d'ancrage des débats et controverses scientifiques récents sur l'émergence, à travers l'urban underclass, d'une nouvelle forme de pauvreté aux Etats-Unis - et maintenant chez nous, d'autant plus que cet ouvrage vient d'être traduit en français (Les oubliés de l'Amérique, Paris, Desclée de Brouwer, 1994, 327 g.)

3 W. Wilson, Les oubliés de l'Amérique, op. cité, p. 33.

4 J. Ion, Le travail social à l'épreuve du territoire, Toulouse, Privat, 1990, p. 39.

5 D. Leborgne \& A. Lipietz, "Idées fausses et questions ouvertes de l'après-fordisme", Espaces et sociétés, n66-67, 1992, p. 63.

${ }^{6}$ O. Claval, "les idéologies spatiales", Cahiers de géographie du Québec, vol. 29, nº 77, septembre 1985, p. 264.
} 


\section{Nouvelle pauvreté et gestion territorialisée de l'exclusion.}

La diversification des situations de pauvreté, l'accroissement du nombre des usagers de l'action sociale et la montée concomitante des dépenses sociales, les insuffisances de modes d'intervention trop segmentés, le contexte de décentralisation ont, en France, favorisé la mise en place d'un nouveau modèle d'intervention sur le social qui a pu être qualifié de "gestion territorialisée de l'exclusion" ". Révolutionnaire en ce sens où il suppose inégalité de traitement selon le lieu, ce modèle d'intervention implique un partenariat entre tous ceux sur qui repose l'impératif national d'insertion inscrit dans l'article premier de la loi du $1^{\text {er }}$ décembre 1988 instituant le RMI. Le recours au territoire comme mode de gestion, qui peut être interprété de prime abord comme une preuve de la reconnaissance par l'Etat de la diversité socio-économique du territoire national, et donc des inégalités spatiales dans les aptitudes à l'insertion et à l'intégration par l'emploi, s'explique aussi par la nécessité pour l'Etat d'obtenir la mobilisation de l'échelon local dans la lutte contre l'exclusion.

Les nouveaux dispositifs d'intervention sociale créés pour parvenir à une nouvelle structuration du lien social reposent sur le principe d'une discrimination positive en faveur des endroits qui cristallisent les problèmes sociaux. Cette idée est novatrice dans notre pays où, contrairement à d'autres dans lesquels l'action sociale prend une forme beaucoup plus communautaire, les outils d'intégration sociale sont traditionnellement à dominante individuelle. Les zones d'éducation prioritaires, les opérations de développement social des quartiers et les dispositifs Bonnemaison de prévention de la délinquance sont des dispositifs territorialisés en ce sens où "ils n'ont pas directement pour objet explicite une clientèle qui serait définie par des caractéristiques d'ayant droit, (...) mais un territoire, ou, plus exactement, l'objet est moins un ensemble d'individus, définis par des critères démographiques ou socioéconomiques, qu'une population localisée. C'est le cadre géographique qui définit la population concernée" 2 .

Les dispositifs relevant de l'insertion professionnelle, dont le RMI, fonctionnent quant à eux "sous le régime de l'aide individuelle et impliquent donc une caractérisation du bénéficiaire (en termes d'âge, de situation professionnelle, de niveau scolaire, etc). Même s'ils (...) nécessitent la création d'instances de concertation établies à des niveaux géographiques qui peuvent être étrangers aux limites administratives et correspondent généralement à la délimitation des bassins d'emplois, ils ne définissent

\footnotetext{
1 J.M. Belorgey, "Réparation et refondation", Territoires, février-mars 1994, p. 41.

2 J. Ion, Le travail social à l'épreuve du territoire, op. cité, p. 39.
} 
pas une population in situ"l. Faut-il pour autant retenir l'hypothèse qu'il ne s'agit pas de mesures territorialisées mais seulement de dispositifs localisés ${ }^{2}$ ? Le RMI fonctionne selon une architecture politico-administrative originale : alors que le bénéfice de l'allocation est régi selon des critères d'ordre individuel, le volet insertion est géré sous la corresponsabilité de l'Etat et des collectivités locales, c'est-à-dire sur des bases inter-partenariales. Si le RMI est une mesure de redistribution en faveur d'une certaine catégorie de personnes, il ne relève pas de la discrimination positive territoriale dans ses modalités de fonctionnement, et ne peut donc compenser par lui-même les différences entre zones riches et zones pauvres. En cela, il ne serait qu'une mesure localisée. Le RMI ne pouvait pas cependant ne pas investir le territoire. Chaque Programme départemental d'insertion (PDI) a supposé une évaluation des besoins en matière d'insertion, compte tenu des caractéristiques locales des bénéficiaires, et un recensement de l'existant de manière à prévoir les moyens à mettre en oeuvre. Les contrats d'insertion sont certes individuels, mais, "à travers ces personnes, ce sont bien les processus locaux d'exclusion et de marginalisation qui sont en jeu, et c'est à partir d'eux que doivent se concevoir les moyens d'insertion ou de réinsertion. C'est donc toute la logique du "territoire" qui est réintroduite ici, logiques de 1'espace rural ou urbain, des bassins d'emploi et des pays, des structures démographiques et des cadres culturels" 3 .

Bien que son cadre d'action soit le département et que le RMI ignore la commune, le législateur a compté sur le dynamisme des pouvoirs locaux et sur la synergie des compétences pour faire du RMI un véritable outil de reconstruction du lien social. Et, parce que, sur le terrain, les différents dispositifs se superposent largement et que la différenciation entre insertion économique et insertion sociale ne résiste pas à un examen approfondi, la territorialisation s'est imposée au RMI. Même si le RMI s'applique de manière égalitaire à l'ensemble du territoire national, le consensus qui a entouré sa mise en place est intrinsèquement lié à un effet de nombre, c'est-à-dire à la menace sociale que représente la constitution de poches de pauvreté dans les quartiers d'habitat social et dans certaines communes de banlieue. Parce que, d'une part, la géographie du RMI est ce qu'elle est, et que, d'autre part, la plupart des dispositifs sociaux territorialisés sont mis en oeuvre dans les quartiers d'habitat social les plus déqualifiés, l'un et l'autre visent de fait "les mêmes populations, touchées par la crise économique, sans espoir de mobilité, souvent mais non exclusivement à forte proportion d'origine immigrée"4

Confrontés quotidiennement aux problèmes posés par l'évolution quantitative et qualitative de la pauvreté, certains acteurs locaux, dans leur commune et leur département, se sont précocement engagés

1 Idem, p. 30.

2 Hypothèse avancée pour le RMI par les auteurs de le RMI à l'épreuve des faits, Syros-Alternatives, 1991, p. 115.

3 R. Lafore, "Les trois défis du RMI. A propos de la loi du 1er décembre 1988", L'actualité juridique - Droit administratif, 20 octobre 1989, pp. 563-585.

4 J. Ion, Le travail social à l'épreuve du territoier, op. cité, p. 34. 
dans la recherche de solutions. Alors que la loi du $1^{\mathrm{er}}$ décembre 1988 a laissé au département le soin de conduire une politique d'allocation des ressources en fonction des disparités de besoins ressenties localement ${ }^{1}$, il apparaît intolérable qu'un traitement inégal des citoyens puisse être la conséquence de la variation des degrés de mobilisation locale. Or la décentralisation a fait courir ce risque. Les nations où l'existence d'une marge de manoeuvre des pouvoirs locaux dans la régulation sociale est ancienne nous montrent en effet la diversité des réponses ${ }^{2}$. Des conditions économiques et des contextes politicoculturels locaux plus ou moins favorables à l'insertion ont abouti de fait à d'importants écarts dans la lutte contre l'exclusion.

La typologie des commissions locales d'insertion en fonction des conditions de fonctionnement proposée par J.-P. Augustin place la dimension territoriale au coeur du dispositif d'insertion : la distinction entre "dynamisme territorial" et "inertie territoriale" infirme l'idée souvent admise que là où "les pauvres sont parmi les riches", les dispositifs d'insertion sont plus nombreux et plus territorialisés, et que, là où "les pauvres sont parmi les pauvres", les réseaux sociaux ne sont pas alimentés ${ }^{3}$. L'exemple de la Haute-Garonne, caractérisée par une dynamique technopolitaine qui obère fortement lès possibilités d'accès à l'emploi et au logement des "plus démunis", par l'opposition politique entre Toulouse et le Département, par l'absence de réelle culture locale en matière d'intervention sociale, a montré qu'il n'en était rien ${ }^{4}$. En ce sens, la mise en oeuvre du RMI à Toulouse et en Haute-Garonne s'est faite dans un contexte inverse à celui de Rennes et de l'Ille-et-Vilaine où ont été expérimentés le Revenu minimum familial garanti (1985-1986), les Travaux d'intérêt collectif (1986), le Complément local de ressources (1986-1988), précurseurs du RMI et de l'insertion. On comprend alors que, après le $1^{\text {er }}$ décembre 1988, la Ville et le Département "se sont immédiatement saisis de la loi sur le RMI et se sont appliqués à rendre opérationnel le I tant controversé du RMI. Les liens peu à peu noués du partenariat, la mise au point d'outils communs, les expérimentations successives ont permis d'aller très vite : les partenaires de l'insertion étaient en bonne partie repérés, les pratiques avaient été soumises à la réflexion, les habitudes de travail collectif étaient prises" ${ }^{5}$. De même, en Mayenne, la mobilisation

\footnotetext{
${ }^{1}$ Le RMI à l'épreuve des faits, op. cité, p. 87.

2 Cf. par exemple, pour les Etats-Unis, J.P. Jones \& J. Kodras, "Restructured regions and families : the feminization of poverty in the U.S.", Ann of the Am. Geogra., 1991, vol. 80, n², pp. 163-183; J. Kodras, "Labor market and policy constraints on the work disincentive effect of welfare", Ann of the Ass. of Am. Geogra., 1986, vol. 76, $\mathrm{n}^{\circ}$ 2, pp. 228-246 ; J. Kodras, J.P. Jones, K. Falconer, "Contextualizing welfares"s work disincentive : the case of female-headed family poverty", Geographical analysis, vol. $26, \mathrm{n}^{\circ} 4$, october 1994, pp. 285-299. Pour l'Allemagne, R. Neef, "The new poverty and local government social policies : a west german perspective", Intern Journal of Urvban and Regional Research, 1992, vol. 16, $\mathrm{n}^{\circ} 2$, pp. 202-221. Pour le Royaume-Uni, M. Goodwin, S. Halford, "Regulation theory, the local state, and the transition of urban politics", Society and space, 1993, pp. 67-88.

3 S. Rosenberg, "Les territoires de l'insertion", dans le RMI à l'épreuve des faits, op. cité, p. 189.

${ }^{4}$ M.C. Jaillet et al, La mise en oeuvre du RMI dans le département de la Haute-Garonne, Rapport de recherche au Plan Urbain, mars $1991,149 \mathrm{p}$.

5 M. Verdié, Voyage à l'intérieur du RMI : l'expérience de Rennes, Syros-Alternatives, 1992, 157 p.
} 
pour l'insertion est relativement plus forte là où les conditions économiques sont a priori les moins favorables, c'est-à-dire dans les campagnes du nord et de l'est du département ${ }^{1}$.

De soutien aux politiques publiques de réduction des inégalités sociales et des écarts de développement du territoire national, l'intervention sociale est passée à un rôle de remède dans le traitement des fractures sociales et territoriales. Les nombreux dispositifs d'intervention décidés dans ce contexte ont en commun de répondre à une logique d'action transversale, établie sur des bases territoriales et partenariales, dans le cadre d'une gestion décentralisée qui élargit le champ de compétences des collectivités locales. La nation se fait alors plus sensible à la diversité de son espace, tant à l'amont, dans la production des processus d'exclusion, qu'à l'aval, dans leur gestion. C'est que les territoires de l'intervention sociale transversale, parce qu'ils sont espace et population, ne sont pas seulement objet d'action mais aussi et surtout moyens d'action de la politique sociale territorialisée. Sur le fond, la prise en compte de la diversité de l'espace par le social et la nécessité de concentrer les moyens en certains lieux ne font qu' "entériner l'échec de la régulation macro-sociale et le retour au micro-social qui seul apparaît comme producteur à la fois de sens, d'identité, et donc d'efficacité"2.

\section{Le territoire local au coeur de la régulation sociale.}

L'irruption généralisée de la référence au local ${ }^{3}$ qui a accompagné le passage d'une gestion sectorielle centralisée du social à une gestion territorialisée et décentralisée est à interpréter au regard de la mondialisation de la sphère économique. Le niveau de l'Etat-nation, qui constituait "la pièce maîtresse de la gestion keynésienne du social et la clé de voûte de la régulation", n'apparaît plus en mesure de répondre "aux pressions du capitalisme mondial et de maintenir l'adhésion des citoyens au

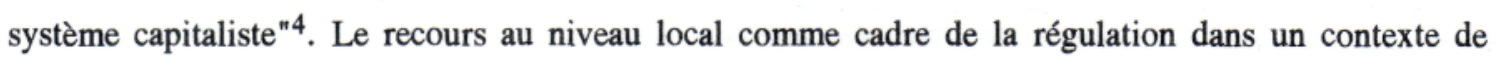
crise du lien social et de fragmentation marque alors le passage à une régulation postkeynésienne qui se caractériserait par une "combinaison encadrement-autonomie"5 où l'entraide de voisinage servirait de justification à l'idéologie communautaire dont on dit qu'elle doit favoriser l'accès à l'autonomie. Dans

\footnotetext{
${ }^{1}$ R. Séchet, "Difficultés et limites de l'insertion en milieu rural : les bénéficiaires du RMI en Mayenne", Norois, vol. 41, $1994 ; \mathrm{n}^{\circ}$ 162 ; pp. 339-352.

2 R. Lafore, "Les trois défis du RMI", article cité, p. 579.

3 Je reprends ici la définition proposée par C. Gagnon \& J.L. Klein : "la notion de local désigne ici des microterritoires qui constituent des milieux de vie et de travail, provoquant chez les communautés des sentiments d'identité et d'appartenance. Les dimensions de ce type de territoire varient d'un contexte national à un autre. Aussi faut-il voir le local comme un cadre spatial de cohésion sociale et non comme un espace déterminé, de nature administrative ou autre". In "Le partenariat dans le développement local : tendances actuelles et perspectives de changement social", Cahiers de géographie du Québec, septembre 1991, p. 252.

4 J. L. KLein, "Les limites de la régulation : crise de l'Etat-nation et gestion du local", Espaces-Temps, 1990, n 43-44, pp. 50-54.

5 Idem, p. 54.
} 
la réalité, elle semble davantage justifier l'établissement de régulations à base territoriale pensées comme moyen de rétablir la normalité et l'ordre urbains.

Quoi qu'il en soit de la solidarité communautaire et de l'entraide entre frères de misère, il faut se prémunir contre tout excès d'optimisme sur les vertus intégratrices du voisinage et des réseaux de proximité. Le soutien économique qui assure la survie est une adaptation à la pauvreté; il n'est pas une véritable résistance contre les processus "exclugènes" et ne signifie pas insertion en ce sens où il ne donne pas accès à la parole ${ }^{1}$ et n'élimine pas les tendances anomiques qui découlent du filtrage et de l'homogénéisation sociale ${ }^{2}$. D. Wilson émet l'hypothèse que l'espace, constituant de l'identité sociale des groupes sociaux, contribue, via les processus cognitifs et la quotidienneté (everyday-life), à soutenir le développement inégal et le déclin des inner cities. L'effet débilitant de la culture de pauvreté résignation, fatalisme - , renforcé par l'homogénéité sociale croissante de la population provoquerait un manque d'intérêt pour la vie publique ${ }^{3}$.

Ainsi, les habitants de Fountain Square à Indianapolis et de Near West Side à Chicago ${ }^{4}$ ont accepté la rhétorique du renouveau favorable à la gentrification et n'ont pas su ou pu développer de stratégie de développement local endogène : la liveable city vision ${ }^{5}$ l'a emporté sur la resident-based vision. Le constat de D. Wilson et J. Browning ("Nous n'avons pas été surpris par l'incapacité de cette population à contrôler les changements dans son quartier dans la mesure où il est bien connu que les plus pauvres et les plus isolés socialement sont les moins aptes à maîtriser leur avenir. Ce qui nous a surpris, ce furent les difficultés que cette population avait à accomplir les actes politiques élémentaires

\footnotetext{
1 "Peut-être davantage que l'accès à l'emploi, même si c'est la demande fomulée par presque tous les bénéficiaires (du RMI), l'insertion est d'abord un accès à la parole". M. Autès, "Les paradoxes de l'insertion", in R. Castel \& J.F. Laé (Dir.), Le revenu minimum d'insersion, une dette sociale, l'Harmattan, 1992, p. 117.

2 Cf l'importance de ce thème dans l'analyse de l'urban underclass par William Wilson ("Public policy research and The Truly disadvantaged", in C. Jenks \& P. Peterson (Ed.), The urban underclass, Washington D.C., The Brookings Institution, 1991, pp. 460480) et dans celle de la relégation par J.M. Delarue (Banlieues en difficultés : la relégation, Syros-Alternatives, 1991).

3 "The political climate in the Near West Side had changed by 1980 . Whereas activism and political dissent had once characterized the area, the influence of exacerbated impoverishment in an era of emerging conservatism helped produce a pervasive community lethargy (...) Intense poverty had a debilitating effect on residents." D. Wilson \& J. Browning, "Politics and community : Chicago's Vear West Side black underclass", Tijdschrift voor Econ. en Soc. geografie, 1994, vol. 85, $\mathrm{n}^{\circ} 1$, pp. 53-66. De son côté, J.M. Delarue note à propos des "banlieues en difficultés" : "Les habitants ne s'estiment guère habilités à entrer dans des querelles de nature politique, y compris lorsqu'elles ont un rapport avec leur propre existence (...) L'impuissance des jeunes à trouver des emplois, celle de la police à mettre fin au trafic de drogue, ou du bailleur à rendre les logements moins sonores, l'impuissance par conséquent de porter des revendications qui soient satisfaites, c'est cela que traduit le déclin du politique". Banlieues en difficultés..., op. cité, p. 43.

${ }^{4}$ Respectivement étudiés dans "Everiday life, spatiality and inner city disinvestment in a US city" (International Journal of Urban and Regional Research, Vol. 17, $\mathrm{n}^{\circ}$ 4, december 1993, pp. 578-594) et dans D. Wilson \& J. Browning, "Politics and community...", ces deux quartiers ont en commun une localisation en inner-city, des taux de pauvreté supérieurs au niveau moyen local et connaissent des processus de gentrification.

5 "Liveable city growth, a term coined by Ley ("Liberal ideology and the postindustrial city", Ann of the Ass. of Am. Geogr., pp. 238258), refers to development premised on aesthetic "revival", which tends to benefit upper income group populations. Restructuring here favours things such as development of the arts, aesthetic enhancement and historic preservation. Liveable city growth is the most prominent form of North American affluent restructuring, and was a term in Indianapolis which spradically appeared in the public discourse about city growth" D. Wilson, "Everiday life, spatiality...", article cité, p. 584, note 3.
} 
qui lui auraient ouvert la possibilité d'une véritable participation politique"1) permet de mesurer la portée intégratrice d'actions visant à favoriser, par la promotion du local et par le partenariat, la réémergence d'une capacité de proposition, c'est-à-dire l'autonomie.

La référence au local, qui relevait, dans les années soixante-dix, du slogan identitaire, régionaliste, voire autonomiste, ressortit maintenant du simulacre ${ }^{2}$. Aujourd'hui, l'Etat-central a recours au territoire local pour mobiliser les élus et l'ensemble des acteurs locaux dans l'application des dispositifs sociaux territorialisés et dans le passage à la transversalité, mais aussi pour inscrire la lutte contre l'exclusion dans un territoire supposé pouvoir générer, à partir de ses ressources propres, de l'insertion et du lien social.

En milieu urbain, le développement social apparaît comme le meilleur moyen de gestion du "découplage" entre la société et "ses composantes périphériques ou décrochées", et donc aussi entre "fractions territoriales"3. Quelques exemples suffiraient à montrer la diversité des nouvelles formes d'intervention / animation sociale dans les "zones" et les "secteurs" que l'on ne veut pas "voir "dériver" à l'américaine et devenir des quartiers de "dépacification sociale"4 ${ }^{4}$ Diverses expériences dans les domaines des transports en commun ${ }^{5}$, des pratiques sportives ${ }^{6}$, des nouvelles formes d'action culturelle $^{7}$ renvoient toutes au principe de l'îlotage, qui s'appuie sur le passage à "une logique dans laquelle le référent $n$ 'est plus la diversité sociale à l'échelle de la ville mais un territoire physique : celui du quartier ou du grand ensemble"8.

Michel Autès a emprunté à Michel Foucault pour montrer comment "le passage du mode de gestion catégoriel des politiques sociales, référé à la sphère du travail, au mode de gestion territorial, référé à la citoyenneté, est, en même temps, un passage d'un mode de gestion disciplinaire à un mode de gestion sécuritaire"9. Pendant la période de croissance, désigner les "pauvres résiduels" comme responsables de leur situation et les rendre plus visibles par leur regroupement dans des cités de transit relevait du mode de gestion disciplinaire qui "se propose de gérer des écarts à la norme. Stigmatisant ces écarts, il les organise et valide la norme, en l'occurrence celle du travail, de la productivité et de

1 "Politics and community...", p. 64. Traduit par moi-même.

2 E. Soja, "Aménager dans / pour la post-modernité", Espaces et Sociétés, n 74-75, 1993, p. 209, d'après J. Baudrillard, Simulacres et simulations, Paris, Galilée, 1981.

3 J.M. Belorgey, "Réparation et refondation", article cité, p. 41.

4 T. Spector, "Transports et exclusion", territoires, février 1993, p. 55.

5 F. Nung \& M. Weughe, "Les agents d'ambiance de Chanteloup-les-Vignes", Territoires, février 1993, pp. 58-60.

6 J.P. Augustin, "Relégation urbaine et pratiques sportives", Sciences de la société, 1993, n 30, pp. $213-217$.

7 A. Lefebvre, "Le "social-culturel" et la requalification des espaces urbains périphériques", dans C. Carreras, G. Jalabert, C. Thouzelier, Restructurations urbaines, 1994, pp. 321-329; J. Palard, "Stratégie politique, action culturelle et intégration socio-spatiale", Sciences de la société, $\mathrm{n}^{\circ} 31,1994$, pp. 5-16.

8 A. Lefebvre, "Le "social-culturel" et la requalification...", article cité, p. 327.

9 M. Autès, "Le territoire, un nouveau mode de gestion des populations", dans Le RMI à l'épreuve des faits, op. cité, pp. 197-213. Les concepts de gestion disciplinaire et de gestion sécuritaire ont été développés par Michel Foucault dans ses cours des années 1978

(Sécurité, territoire, population) et 1979 (Naissance de la biopolitique). 
l'efficience"1. Dans le mode de gestion sécuritaire, il s'agit davantage de "rationaliser les problèmes posés à la pratique gouvernementale par les phénomènes propres à un ensemble de vivants constitués en population" ${ }^{2}$, de faire que, malgré la pauvreté et l'exclusion, on puisse vivre dans nos villes. Dans ce mode de gestion où l'on calcule des coûts et des niveaux de tolérance, "il revient au gouvernement non seulement d'organiser son pouvoir, mais aussi d'agir à l'articulation du milieu et de l'espèce humaine ${ }^{n 3}$. Alors, politiques urbaines et politiques sociales s'imbriquent pour fixer des marginalités qui pourraient menacer l'ordre social et le pouvoir.

Le renforcement du sentiment d'appartenance communautaire peut apparaître comme un préalable à la réussite de toute opération de développement social urbain. La communauté qui procure des services et de l'entraide, c'est-à-dire de la survie, est aussi supposée, par le jeu des interactions, contribuer à donner du sens aux lieux et créer de la territorialité. On voit bien alors l'intérêt du passage à une intervention sociale transversale et territorialisée supposée favoriser l'émergence d'un projet local commun. Le monde rural, ou du moins sa représentation, sert ici de modèle idéologique à la gestion culturelle et identitaire des nouvelles régulations sociales: "Si l'espace rural ressortit encore à l'aménagement, l'espace urbain ressortit de plus en plus à la solidarité - inversion intéressante d'autant que, dans les quartiers d'habitat social, le modèle du village fera référence pour ceux qui souhaitent restaurer les solidarités informelles ${ }^{4}$. " Dans la ville, le "village" devient "quartier", support d'une communauté mythique à finalité d'apaisement social.

Le couple autonomie / encadrement renvoie à "deux versions possibles" de la gestion territoriale du social : dans un sens, la vie associative est valorisée, et territorialiser signifie "multiplier les espaces de parole, les lieux de rencontre entre acteurs sociaux, les objets de négociation"; dans la version administrative, c'est "opérer directement sur la gestion de la norme et non seulement intervenir sur les écarts" ${ }^{5}$. Cette gestion de la norme, qui est plus ou moins explicite dans les discours et les actions politiques, a ramené au premier plan la question du rôle de l'espace dans le contrôle social ${ }^{6}$. Et c'est précisément lorsque les transgressions à ce qui apparaît comme normal sont redoutées parce que potentielles que la mise en action de la norme urbaine devient une des priorités du pouvoir local. Si la coercition et l'encadrement par l'îlotage dominent au niveau du quartier, à un niveau supérieur ce sont les pratiques d'adhésion identitaire - le sens commun - qui priment.

\footnotetext{
1 M. Autès, "Le territoire, un nouveau mode de gestion ...", texte cité, p. 208.

2 M. Foucault, Résumé des cours, Julliard, 1989, p. 109 (1979 : Naissance de la biopolitique).

3 M. Autès, "Le territoire, un nouveau mode de gestion...", texte cité, p. 208.

${ }^{4}$ Le RMI à l'épreuve des faits, op. cité, p. 84. Souligné par l'auteur.

5 M. Autès, "Les paradoxes de l'insertion", texte cité, p. 119.

6 D. Loschak, "Espace et contrôle social", dans J. Chevalier et al, Centre, périphérique, territoire, Paris, PUF, 1978, p. 154 et 157.
} 
La prise en main du social et de l'image de la ville atteint une importance toute particulière dans les villes qui, comme Rennes ou Montpellier, ont plutôt fait le pari d'un développement technopolitain élitiste, et ont dû se doter de projets particulièrement raffinés et sophistiqués pour concilier modernité et solidarité. L'orientation technopolitaine, fondée sur la "valorisation de la matière grise, de l'intelligence, l'innovation, la créativité, la facilité d'adaptation, l'esprit d'entreprise" ${ }^{n 1}$, s'accompagne dans les propos, si ce n'est dans l'esprit des élus rennais, d'une préoccupation de progrès social. De fait, Rennes a innové dans ce domaine. La gestion des contradictions était beaucoup plus difficile à Montpellier ${ }^{2}$ qui n'a pas le passé de Rennes en matière d'engagement dans le social, où la thématique de la modernité a été poussée beaucoup plus loin, où les niveaux de chômage, de pauvreté, de délinquance sont le reflet de fortes segmentations sociales. Alors, après avoir été la "Surdouée" (1985), Montpellier est devenue "Cité coeur" : "La solidarité est donc assignée comme contre-feu de la logique des technopoles. Mieux, par un renversement dont les politiques ont le secret, la Technopole est maintenant affichée comme un "formidable instrument de solidarité" (février 1993)"3. La "mise en scène de l'identité [et du lien social qui la sous-tend] et de la citoyenneté urbaine" ${ }^{4}$ à travers la redécouverte du patrimoine, les politiques d'image, les commémorations festives, sont aujourd'hui des composantes majeures de la création d'urbanité5.

Les modèles de villes qui apparaissent dans les discours nous renseignent moins sur l'espace que sur la société, sur ses échecs, sur les valeurs, intérêts et stratégies de ceux qui contrôlent les représentations de $1^{\prime}$ espace ${ }^{6}$. La mobilisation autour du culturel joue à ce niveau de la réarticulation des espaces d'exclusion avec l'ensemble du corps social. A Tours7, à Mexico ${ }^{8}$, à Barcelone, "méfions-nous du déploiement intéressé de certains arguments écologiques en faveur d'une ville habitable, car l'assainissement de l'environnement [la revalorisation du centre-ville], appliqué avec des critères discriminatoires, divise la métropole en zones propres et sales" et constitue un facteur-clé de différenciation sociale et de renforcement de la dualisation de la ville ${ }^{9}$. Face à la solidification des poches de pauvreté qui instaure une situation conflictuelle, la "guerre à la pauvreté" a pour objectif "d'assurer la protection et l'administration des parties dynamiques et innovatrices de la métropole, en

1 Projet pour l'agglomération rennaise, Rennes-District, 1993.

2 A. Marchand, "Le social saisi par le discours politique. L'exemple de Montpellier", Sciences de la société,n ${ }^{\circ} 31,1994$, pp. 17-34.

3 Idem, p. 21.

4 I. Garat, "vivre Bayonne intensément. Mise en scène de l'identité et de la citoyenneté urbaine à travers la fête", Sciences de la société, $\mathrm{n}^{\circ} 31,1994$, pp. 109-124.

5 M. Roncayolo, La ville et ses territoires, Folio-Essais, Gallimard, 1990, p. 89.

6 J. Monnet, La ville et son double. La parabole de Mexico, Nathan, coll. Essais et recherches, 1993, p. 14.

7 M. Lussault, Tours ; images de la ville et politique urbaine, Maison des Sciences de la Ville, Université François Rabelais, Tours, Collection Sciences de la Ville $\mathrm{n}^{\circ}$ 3, 1993, $415 \mathrm{p}$.

8 J. Monnet, La ville et son double..., op. cité.

${ }^{9}$ P. López, "Ville entreprise et dualité sociale dans la Barcelone olympique", dans CIEU-Toulouse, Barcelone-Toulouse, horizon 2000,1993 , p. 168. 
évitant tout contact avec les aires déprimées. Ces dernières feront l'objet d'une politique de gestion des risques avec, dans le domaine de l'assistance sociale, une intervention minimale mais suffisante pour garantir le contrôle social" ${ }^{1}$.

D'autre part, "le spectre diffus de l'insécurité urbaine est un atout utilisé pour orchestrer le consensus au sein de la ville" dans la mesure où, en désignant certains groupes et quartiers, il consolide la peur de l'autre et favorise l'intériorisation de l'ordre urbain : "La guerre à l'indiscipline, comme au début du siècle, ne sert plus seulement à véhiculer le consensus. Sa mission est aussi de s'attaquer au dissentiment. L'administration de la métropole exige d'anéantir tout signe d'antagonisme diffus ou déclaré. Autrement dit, il s'agit de rendre impensables et impossibles les autres manières de vivre la ville"2. Sur le même thème, T. Cresswell suggère qu'à travers l'importance des moyens engagés par la ville de New York pour lutter contre les graffitis, la préoccupation est moins la propreté des buildings que New York elle-même comme symbole de contrôle, d'ordre, d'harmonie, c'est-à-dire le rétablissement de la norme (en tant qu'occupation du temps et pratiques de l'espace conformes au sens commun) $)^{3}$.

\section{De l'exclusion au contre-territoire.}

A chacun des deux niveaux - le quartier et la ville -, la gestion du lien social prend la forme de mécanismes de "reconstitution horizontale d'une société en apparence éclatée sous l'effet de la crise de l'Etat nation", et l'intervention sociale (y compris quand elle passe par le soutien à la pratique sportive ou par l'offre culturelle) est une démarche où "la proximité territoriale prime sur les distances sociales $^{\text {4 }}$. La territorialisation des dispositifs sociaux ne va pas sans effets pervers car la combinaison encadrement/ autonomie constitutive de la régulation post-keynésienne débouche sur le couple effacement / stigmatisation. Alors que la volonté politique est d'inverser la spirale de la dégradation et de la marginalisation, des pratiques trop spécifiques peuvent contribuer à un marquage négatif de l'espace, et ainsi renforcer l'exclusion et favoriser le racisme, mais aussi la prise de conscience territoriale - et ethnique - dans les populations des espaces d'intervention. Pour les catégories sociales confrontées à l'exclusion et à la pauvreté, la conscience territoriale aboutit à des phénomènes d'isolement, de clôture, de rupture, selon qu'elles optent pour "l'auto-effacement (pauvre-honteux)", la

\footnotetext{
1 Idem, p. 170

2 Ibid, p. 171.

3 T. Cresswell, "The crucial "where" of graffiti : a geographical analysis of reactions to graffiti in New York", Society and Space, 1992, pp. 329-344.

4 C. Cagnon \& J.L. Klein, "Le partenariat dans le développement local...", article cité, p. 243.
} 
"constitution de micro-sociétés (ghettos)" ou encore la "mise en scène ostentatoire de la souffrance individuelle" ${ }^{n 1}$.

L' "insoumission" et la "rébellion" 2 des périphéries urbaines peuvent être perçues comme des actes positifs de résistance et de refus de la soumission au "fantasme médiatique" 3 : "Il y a une profonde différence entre "être participés" et participer. Cela introduit une distinction entre une participation formelle sur invitation, et une participation informelle par effraction. La première serait aliénée au jeu des majorités et confinée à une circulation dans les rouages institutionnels; la seconde, en revanche, apparaîtrait comme l'expression de flux minoritaires qui, désireux de sortir du piège de la norme dominante, préferent se dérober à la capacité de "phagocytage" par les engrenages institutionnels de l'ordre urbain. [Les pratiques de participation des individus et des groupes sociaux à la vie de la cité] peuvent aussi bien apparaître comme piège (quand on circule sur les chemins prescrits par l'ordre urbain) ou comme dispositif de libération (quand on invente des itinéraires proscrits)" ${ }^{4}$. La marginalité choisie du contre-territoire pourrait donc être interprétée comme l'expression d'une résistance à base territoriale.

La conquête médiatique peut se faire par l'ostentatoire de l'autodestruction, de la mendicité et de l'agressivité. Aux parcours individuels et aux regroupements instables de jeunes qui exposent leur "galère" au centre des villes 5 , se surimpose la stabilité des "bandes identitaires" organisées en réseaux, et dont "la violence délimite territoires et espaces sociaux; ritualisée dans le pillage et la "casse", l'attentatoire, elle vise à forcer la reconnaissance sociale à travers les yeux de la police, des médias"6. Quand les dispositifs territorialisés se superposent dans des quartiers à forte proportion de population d'origine étrangère, l'expression "milieux défavorisés" n'est souvent qu'un euphémisme pour "jeunes issus de l'immigration"7. Les bandes sont alors à forte composante immigrée et les actes de révolte favorisent le déplacement de la question urbaine vers la question ethnique. Dans la mesure où "toute identité ethnique minoritaire, qu'elle soit fondée sur un critère de territoire, de langue, de religion, de race ou même de lignage, est en grande partie assignée par le groupe majoritaire dont le regard est, d'une certaine manière, constituant du groupe minoritaire" 8 , les transformations des marchés de

${ }^{1}$ A. Marchand, "Le social saisi par le discours politique", article cité, p. 29.

2 P. López Sanchez, "Barcelona 1992, la requisa de una metrópoli. Anotaciones para una geografia política de lo social en la metrópoli", dans C. Carreras, G. Jalabert, C. Thouzelier, Restructurations urbaines, CIEU, Toulouse, 1994, p. 233 et suiv.

${ }^{3}$ M. Fourdin, "Communication urbaine : 1'apport des représentations sociales", IUT Toulouse, Sciences de la société, $\mathrm{n}^{\circ} 30$, octobre 1993, p. 155.

${ }_{5}^{4}$ P. López Sanchez, "Barcelona 1992...", texte cité, p. 234.

5 A. Penven, "Précarité et exclusion... Les ambiguités du traitement social des crises urbaines" Travaux et documents de l'URA 915, $\mathrm{n}^{\circ}$ 1 , juin 1994, p. 30.

6 A. Marchand, "Le social saisi par le discours politique", article cité, p. 30.

7 J. Ion, Le travail social à l'épreuve du territoire, op. cité, p. 34.

8 I. Taboada Leonett, Les immigrés des beaux quartiers. La communauté expagnole dans le XVIe, CIEMI-L'Harmattan, 1987 , p. 30. Souligné par l'auteur. 
l'emploi et des politiques d'immigration, d'une part, la territorialisation des politiques sociales, et la stigmatisation qui peut en découler, de l'autre, cumulent leurs effets pour favoriser les réflexes défensifs de structuration identitaire.

Les effets des transformations économiques pèsent de manière toute particulière sur ceux qui sont confinés dans l'économie informelle ou le second marché du travail. En conséquence, les formes traditionnelles de solidarité horizontale, telles qu'elles pouvaient s'exprimer dans les organisations syndicales à travers l'identité ouvrière, perdent de leur influence pendant que se développent de nouvelles solidarités davantage basées sur les liens ethniques ou communautaires ${ }^{1}$. La délimitation de territoires-cibles, leur confusion avec des populations-cibles, n'est pas non plus sans risques: "L'ethnicisation de l'intervention sociale (...) induit le séparatisme ethnique alors que le problème des familles en grande détresse n'a rien d'ethnique : il est d'abord économique et social. Elle suppose que les jeunes d'origine étrangère constituent un groupe structuré : ils sont au contraire, complètement dispersés et souvent ignorants 1'un de 1'autre"2. Les nombreux dispositifs territorialisés, en projetant une image communautaire sur une communauté qui n'existe pas, favorisent donc l'ethnicisation des banlieues et un double processus de construction de frontières symboliques et de réponse sécuritaire.

Ce phénomène de clôture sur des bases ethniques, revers d'un repli sur le local et la communauté, pourrait d'ailleurs bien être une tendance lourde dans les sociétés contemporaines, la clôture identitaire générant des réflexes de défense territoriale contre des minorités rivales menaçantes, comme dans le cas des émeutes de 1992 à Los Angeles ${ }^{3}$, alors que là où l'organisation interne de la ville impose le partage d'un même espace par les gagnants et les perdants, la peur du crime et de la révolte donne naissance au "grillage de l'exclusion"4, mais aussi à une "architecture de bunker"5. Cela ressemble fort à une inversion de l'enfermement d'autrefois : à défaut de pouvoir isoler les pauvres du reste de la société, ce sont les riches qui se coupent d'eux.

\footnotetext{
${ }^{1}$ E. Pugliese, "Restructuring of the labour market and the role of Third World migrations in Europe", Society and Space, 1993, vol. 11, p. 521.

2 H. Vieillard-Baron, "Des banlieues aux ethnies. Géographie à voir, histoire à suivre...", Les Annales de la recherche urbaine, $\mathrm{n}^{\circ} 64$, 1994, pp. 96-102.

3 C. Ghorra-Gobin, "Los Angeles 1992. La première émeute multi-ethnique aux Etats-Unis", Hérodote, n65-66, 1992, p. 337.

4 "Avec ses tentes rongées par l'humidité, ses bâches trouées tendues sur des ficelles, ses toiles jetées par dessus un banc, le cossu petit parc de Lincoln's Inn Fied est devenu depuis quelques années "Tenteville' (Tent City), un havre pour quelques dizaines des 2500 sansabri de Londres. Le tout dans un quartier huppé, à cinq minutes à pied de la City. (...) Le conseil local, pressé par une association de résidents, a érigé un haut grillage métallique interdisant l'accès - et la sédentarisation - dans toute une partie du jardin. Officiellement, il s'agit de refaire les pelouses et bosquets". dans "Londres : le grillage de l'exclusion", Le Figaro, 16 décembre 1992, p. 38.

5 J. Short, "Yuppies, yuffies and the new urban order" ", Transactions Institute of British Geographers, 1989, vol. 14, pp. 173-188.
} 


\section{Conclusion}

C'est en fonction de l'oubli de la pauvreté relative dans le grand retour de la pauvreté absolue, et de la réorientation des regards d'une problématique inégalitaire vers l'exclusion, qu'ont été élaborées de nouvelles politiques sociales. Les réponses pratiques à la nouvelle situation, et surtout à sa perception, sont faites d'un ensemble de "recettes sociales, économiques, idéologico-culturelles, voire des recettes identitaires" 1 qui sont clairement positionnées à l'aval des processus, et ont pour finalité de gérer des marges de plus en plus étendues et perçues comme une menace.

Le revenu minimum d'insertion apparaît comme la principale "recette" économique adoptée en France. Sa création est à la fois reconnaissance du droit à la subsistance pour tous et conséquence des transformations des marchés de l'emploi dont l'ampleur ôte toute crédibilité à la régulation sociale par le seul jeu de l'économie. Ce contexte, sans doute autant que la volonté d'imposer une contrepartie à l'allocation, explique l'originalité de ce revenu minimum qui est aussi recette sociale. La distinction établie par la loi du $1^{\mathrm{er}}$ décembre 1988 entre insertion professionnelle et insertion sociale a évolué vers une hiérarchisation au sein d'un sous-système, que les uns parent du label de rétablissement de la dignité alors que les autres en appellent aux workhouses anglaises du dix-neuvième siècle pour justifier le recours à la métaphore de l'enfermement, et dont la finalité est inscrite dans le thème, souvent invoqué, du "lien social".

Les fragmentations sociales et spatiales inhérentes aux mutations économiques, le développement des idéologies libérales, les politiques de rigueur budgétaire ont contribué à déconsidérer les "recettes keynésiennes" et l'action régulatrice de l'Etat, et à "poser avec acuité la question du rôle du politique et du partage des prérogatives entre l'Etat, les échelons territoriaux, le secteur privé et les citoyens" ${ }^{2}$. Le territoire a donc été investi par l'action sociale, parce que l'administration a eu besoin de se territorialiser pour gagner en crédibilité et en efficacité après que des modes d'intervention sectoriels trop fragmentés eurent montré leurs insuffisances. Cette territorialisation suppose discrimination positive, c'est-à-dire remise en cause du principe de traitement égalitaire, et mobilisation des acteurs locaux.

Dans une logique sécuritaire de contrôle social, c'est par le territoire que sont mises en oeuvre des recettes idéologiques et culturelles prenant en compte la sociabilité de base qui sert de ciment aux ensembles sociaux. Les acteurs locaux s'appuient sur l'existence de solidarités de voisinage, sur le modèle du développement local communautaire et sur la mise en scène identitaire et patrimoniale pour

\footnotetext{
${ }^{1}$ E. Balibar, "Inégalités, fractionnement social, exclusion. Nouvelles formes de l'antogonisme de classe ?", in J. Affichard \& J.B. de Foucauld, Justice sociale et inégalités, Paris, Editions Esprit, 1992, p. 155.

2 S. Biarez, "Recomposition territoriale et urbaine en Europe", Sciences de la société, n 30, 1993, p. 105.
} 
assurer les micro-régulations. L'utopie territoriale apparaît alors comme un moyen d'enfermer les rapports sociaux dans les bornes du contrôlable" et il arrive que l' "on ne cherche plus à ressusciter les communautés de la société d'hier; on décide d'en fonder de nouvelles"1. Cette invention du local inhérente à la gestion territorialisée du social peut se faire sans lien réel avec l'espace vécu de ceux qu'elle entend contrôler. Elle n'est pas sans risque si, dans un contexte d'assignation à résidence, cette construction identitaire imposée sur des bases locales est transformée en une contre-culture débouchant sur un renforcement de la perte de porosité de l'espace urbain.

Manuscrit reçu en février 1995.

${ }^{1}$ P. Claval, "Les idéologies spatiales", article cité, p. 267 et p. 265. 